\title{
L'utilisation des nouvelles technologies de l'information et de la communication (exemples concrets)
}

\author{
Using new information and communication technologies \\ (concrete examples)
}

par M. Léobet

Mate/DPPR/sous-direction de la prévention des risques majeurs

The ministry of regional planning and environment started to publish on internet information related to the prevention of the major risks. This action complies at the same time with the application of the law of July 22, 1987 and the governmental action plan for the society of information. After several tests in 1998, a "service of publication on internet of the cartographies of risks" intended initially for the prefectures, is in the course for installation.

\section{I 口UN DOUBLE CONTEXTE, LÉGISLATIF ET GOUVERNEMENTAL}

La démarche dont nous allons vous présenter un exemple aujourd'hui est la résultante de deux démarches : le développement à l'initiative du ministère de l'Aménagement du Territoire et de l'Environnement (MATE) de l'information sur les risques majeurs d'une part, et le programme d'action gouvernemental «Préparer l'entrée de la France dans la société de l'information" (PAGSI) d'autre part.

\subsection{L'obligation d'informer sur les risques majeurs}

L'article 21 de la loi du 22 juillet 1987, relative à l'organisation de la sécurité civile, à la protection de la forêt contre l'incendie et à la prévention des risques majeurs précise : «les citoyens ont un droit à l'information sur les risques majeurs auxquels ils sont soumis dans certaines zones du territoire et sur les mesures de sauvegarde qui les concernent. Ce droit s'applique aux risques technologiques et aux risques naturels prévisibles (...)». Son décret d'application du 11 octobre 1990 définit deux niveaux de documents, un dossier synthétique établi par le préfet reprenant la description des risques et les mesures de sauvegarde prévues pour limiter leurs effets, et un document d'information établi par le maire recensant les mesures de sauvegarde répondant aux risques sur le territoire de la commune. Ces documents sont librement consultables en mairie.

Jusqu'en 1998, environ 2000 communes ont fait l'objet d'un dossier synthétique, dit DCS. Plus de 2000 dossiers devraient être réalisés dans la seule année 1999, ce qui témoigne d'une forte accélération de la démarche, aidée par une augmentation significative des budgets, qui sont passés de 6 à $15 \mathrm{MF}$ en 3 ans. Mais ces informations peinent encore à parvenir jusqu'aux citoyens, puisque moins de 200 documents d'information ont été réalisés par les maires.

1.2 Le programme d'action gouvernemental «Préparer l'entrée de la France dans la société de l'information»

Le PAGSI définit, quant à lui, six chantiers prioritaires, dont «la mise des technologies de l'information au service de la modernisation des services publics». Ses deux têtes de chapitre sont «faciliter l'accès des citoyens à l'administration par internet» et «moderniser le fonctionnement de l'Etat».

Le ministère de l'Aménagement du Territoire et de l'Environnement a donc décidé de fournir via internet le plus d'information possible, et en particulier les cartes d'aléas. L'objectif est, par la diffusion de l'information, de développer une culture du risque et de faciliter la mise en place des actions de prévention et d'aménagement.

\section{UUNE COMMUNICATION GRAND PUBLIC}
2.1 La publication du recueil national des communes à risques

Dans le cadre de l'information préventive sur les risques majeurs, presque tous les préfets ont réalisé, entre 1994 et 1998, une analyse des risques dans leur département, commune par commune. Sans doute moins utile qu'une cartogra- 
phie précise des aléas, cela permet néanmoins de disposer enfin d'une vision nationale, agglomérée de la situation. Le résultat a été cartographié, et publié, pour des raisons techniques, à l'adresse www.prim.net. Il montre quelques curiosités, comme l'influence des limites administratives sur les risques, mais il offre l'immense avantage de fournir à chacun une information jusqu'ici quasiment impossible à obtenir, et enrichie de fiches communales reprenant les arrêtés de catastrophe naturelle, par exemple.

\subsection{Le contenu de la communication sur les dossiers synthétiques}

Les dossiers synthétiques présentent donc deux aspects, description des risques et mesures de sauvegarde. Nous nous concentrerons sur celui de la description des risques. Sans que cela ait été explicitement imposé, la cartographie est devenue son media principal. C'est souvent la seule partie qui différencie un dossier synthétique d'un autre, et celle qui attire en premier lieu le lecteur.

Car ce qui intéresse le lecteur, c'est d'abord la situation de l'aléa par rapport à sa situation propre ou celle de ses proches. La communication est donc d'abord spatiale. Destinée au grand public, elle doit prendre une forme culturellement acceptée par le plus grand nombre. C'est pourquoi nous avons choisi comme référentiel les cartes au 1:25000 de l'institut géographique national, en écartant, par exemple, les orthophotographies ou les sorties de bases de données géographiques. A ce titre, nous avons signé une convention avec l'IGN pour acquérir l'ensemble des scannages de ses cartes au 1:25000 et le droit de les diffuser sur internet comme référence cartographique et représentation des enjeux.

Les aléas, à partir de la connaissance historique ou scientifique des phénomènes, sont présentés sous formes de polygones ou, plus rarement, de points. Ils sont tramés afin de laisser apparaitre, par transparence, le fond, et donc la plupart des enjeux menacés. Le risque d'inondation étant le plus courant en France, il est, bien sûr, très bien représenté dans ces documents.

\subsection{Les cartes d'aléas sur Internet}

Nous avons testé, en 1998, sur 5 départements - l'Aude, la Haute-Garonne, la Gironde, le Maine-et-Loire et l'Orne la mise en ligne de cette information. Différentes solutions techniques ont été essayées. Les solutions à base d'images scannées, même compressées dans des formats PDF, n'ont pas donné satisfaction, car trop lentes d'affichage et trop peu pratiques. Par contre, les récents logiciels dédiés à la publication de cartographie sur internet, à base de systèmes d'information géographique, donnent déjà des résultats probants et disposent d'immenses possibilités d'amélioration.

Nous avons choisi de présenter, comme exemple concret de réalisation, l'exemple de la ville de Toulouse. D'abord parce qu'il nous paraît représentatif de ce que nous souhaitons accomplir. Les fonctionnalités, l'interactivité et le niveau de détail accessible aux internautes est à la pointe de ce que la technique peut offrir aujourd'hui. Ensuite, parce qu'il n'est pas neutre que le DCS de Toulouse ait été présenté à ses administrés par le maire, M. Dominique Baudis. Enfin, parce que le travail pilote des services de la préfecture nous a semblé mériter cette mise en avant.
En 1999, tirant enseignement des réussites et des difficultés rencontrées, cette démarche a évolué vers la mise en place d'un véritable «service de publication sur internet des cartographies d'aléas" destiné en premier lieu aux préfectures. Les départements de la Gironde, du Vaucluse et des Vosges sont volontaires pour tester cette démarche nouvelle. Il s'agit de développer, pour les préfectures, un service unique de mise en ligne des données sur la prévention des risques. Les parties les plus complexes sont celles liées à la structuration des données dans un format compatible avec le serveur internet, l'administration des données à publier et la gestion du serveur géographique. Nous offrons un service centralisé de publication sur internet qui prend en charge ces processus. Mais les procédures respectent la part d'autonomie et la responsabilité des préfectures, comme le choix des processus de fabrication des DCS, celui des logiciels de création de l'information, et le choix de la diffusion et de la mise à jour des données. S'il s'agit bien d'une structure informatique centralisée, son alimentation est, par contre, prévue comme étant parfaitement déconcentrée.

La cartographie des zones inondables est également concernée. Elle doit être à l'honneur le deuxième mercredi d'octobre, à l'occasion de la journée nationale de la prévention des risques majeurs que le MATE souhaite instituer. Nous publierons les atlas des plus hautes eaux connues des bassins Oise et Aisne, puis ceux des zones inondables de la région Ile-de-France.

\section{III —CONCLUSION}

Il y a encore un an, la communication via internet était parfois vue comme une mode, étant donné le faible nombre d'internautes en France. Or, au début de 1999, selon la Délégation interministérielle à la réforme de l'Etat, 4,5 millions de Français étaient connectés à Internet, mais $19 \%$ de la population, plus de $30 \%$ de franciliens y avaient accès, à leur domicile ou au bureau, et plus de $70 \%$ des enseignants étaient abonnés.

L'information via internet vise à fournir au citoyen les moyens d'intervenir dans le débat public, à l'occasion des différentes actions de prévention, et à participer au développement d'une culture du risque, qui nous semble la façon la plus efficace, sur le long terme, de faciliter la réduction de la vulnérabilité. Même si internet est réputé comme étant un lieu de «temps réel», cette démarche sera longue. Merci d'avoir accepté l'exposé de ses prémisses.

\section{IV — SITES RELATIFS À CET EXPOSÉ}

- www.caplaser.fr/maquette/pref31/ (adresse temporaire, qui sera remplacée par celle de la préfecture de Toulouse), avec des dossiers synthétiques de villes de Haute-Garonne, dont Toulouse.

- www.prim.net : site présentant les cartes du Recueil national des communes à risques, ainsi une sélection de sites traitants de ce thème.

- www.environnement.gouv.fr/actua/cominfos/dosdir/ DIRPPR/dosdppr.htm : pages présentant les PPR et divers dossiers thématiques.

- www.internet.gouv.fr/francais/index.html : le site du PAGSI.

- www.mtic.pm.gouv.fr : site de la Délégation interministérielle à la réforme de l'Etat. 
Eichier Edtion Alficher Aler Communicsta pide

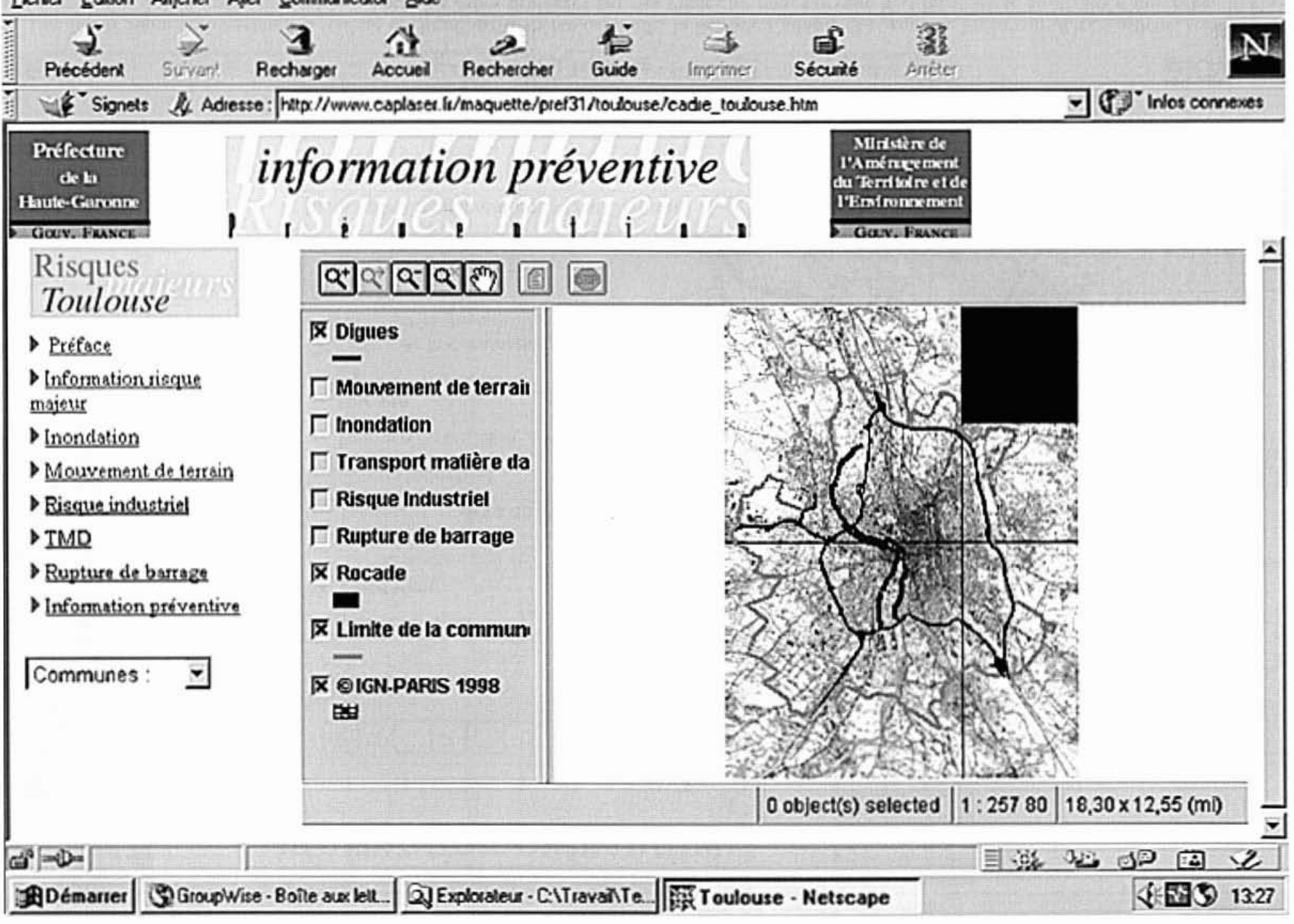

1. Première page du dossier synthétique de Toulouse. L'écran se partage en deux parties. Celle de droite renvoie vers des textes de description des risques et des mesures de précaution à prendre. Un tableau déroulant permet de choisir la commune. Celle de gauche présente, en haut, la barre d'outils : zooms et déplacement manuel dans l'image. A droite, une barre de légende interactive permet l'affichage du ou des thèmes souhaités par simple clic. Au centre, la commune est affichée. 


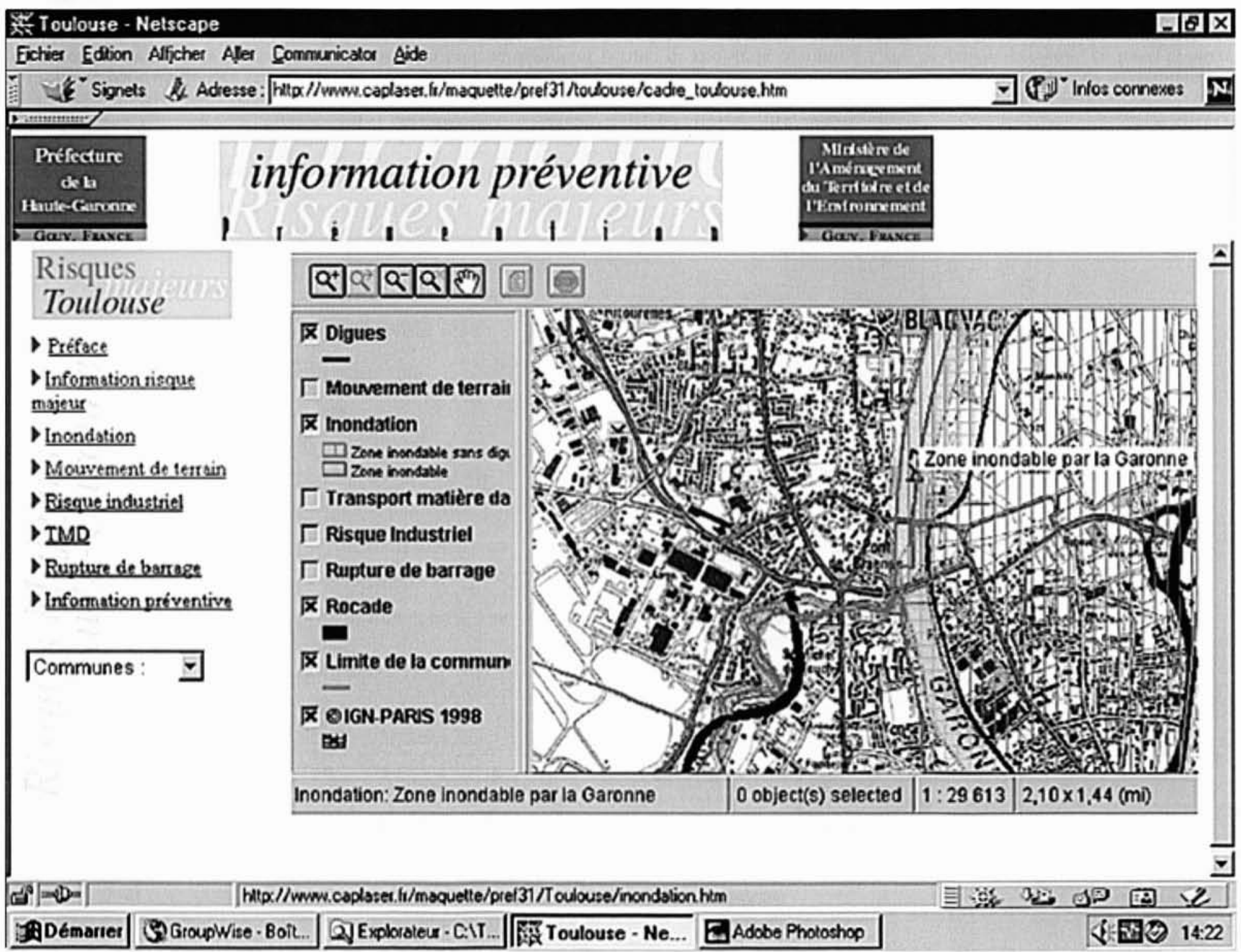

2. Une fois le zoom activé selon son souhait et cliqué sur le thème demandé, la légende s'adapte et la zone de l'aléa considéré s'affiche, selon un ou plusieurs types. Ici, la distinction est faite entre les zones protégées par des digues ou non. Lorsque le curseur passe au-dessus d'une zone, un cartouche explicatif s'affiche. 
Exchier Edtion Alficher Aler Communicalor Aide

\begin{tabular}{|c|c|c|}
\hline$\xi^{*}$ Signets & 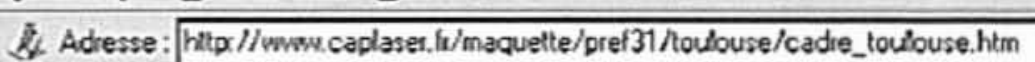 & $\nabla$ P Inlos connexes $\mathrm{N}$ \\
\hline
\end{tabular}

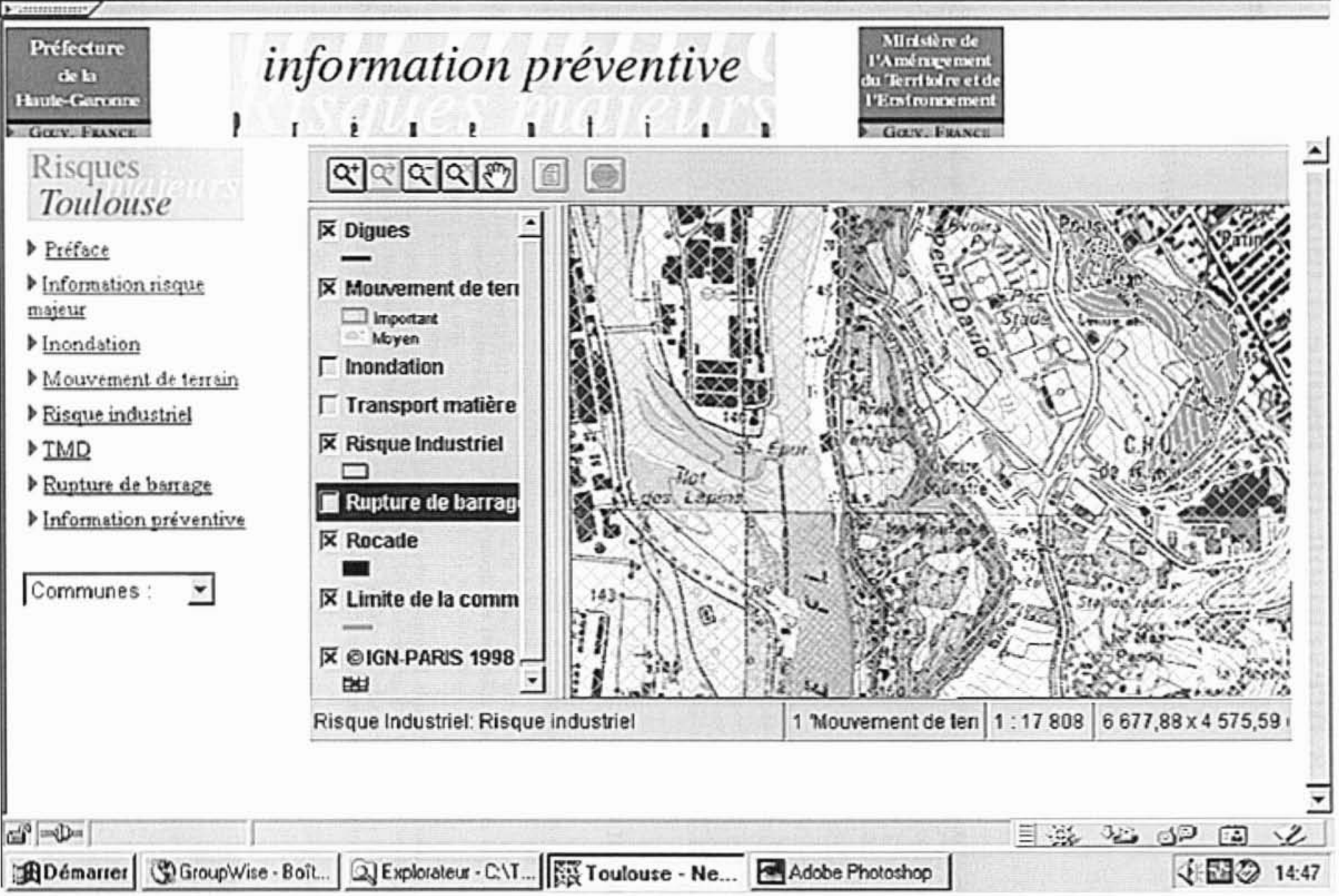

3. Plusieurs thèmes peuvent être affichés simultanément. Le résultat, trop confus pour être utilisé dans le document imprimé, permet ici de comprendre les contraintes sur une zone donnée. Il est possible de sélectionner une zone spécifique pour mieux la faire ressortir. 